\title{
Postconditioning in ST-elevation myocardial infarction: a systematic review, critical appraisal, and meta-analysis of randomized clinical trials
}

This article was published in the following Dove Press journal:

Vascular Health and Risk Management

8 August 2014

Number of times this article has been viewed

\section{Abdelnoor ${ }^{1,2}$ \\ I Sandven' \\ S Limalanathan ${ }^{3}$ \\ J Eritsland ${ }^{3}$}

'Centre of Epidemiology and Biostatistics, ${ }^{2}$ Centre of Clinical Heart Research, ${ }^{3}$ Department of Cardiology, Oslo University Hospital, Ullevål, Norway
Correspondence: M Abdelnoor Centre of Epidemiology and

Biostatistics, Centre of Clinical Heart Research, Oslo University Hospital,

Ullevaal, 0407 Oslo, Norway

Tel +4799540888

Fax +4722118479

Email michelab@online.no
Objective: We aimed to summarize the evidence from randomized clinical trials studies examining the efficacy of ischemic postconditioning (IPost) in ST-elevation myocardial infarction.

Design: The study was a systematic review and critical appraisal, with meta-analysis of randomized clinical trials.

Materials and methods: We searched the literature. A total of 21 randomized clinical trials were identified. Both fixed effect and random effects models were used to synthesize the results of individual studies. Heterogeneity between studies was examined by subgroup and random effects meta-regression analyses, considering ptient-related and study-level variables. Publication bias, or "small-study effect", was evaluated.

Results: Substantial heterogeneity was present. The random effects model pooled estimate for the outcome infarct size assessed by cardiac magnetic resonance was estimated by the standardized mean difference $(\mathrm{SMD})=-0.06,95 \%$ confidence interval $(\mathrm{CI}):-0.34$ to 0.21 , ie, no effect of IPost. For the end point infarct size, estimated by biomarkers of myocardial necrosis, an overall pooled effect was $\mathrm{SMD}=-0.58,95 \% \mathrm{CI}:-0.96$ to -0.19 . This effect disappeared in powered and nonbiased studies ( $\mathrm{SMD}=0.03,95 \% \mathrm{CI}$ : -0.48 to 0.55 ). Finally, for the outcome left ventricular ejection fraction, $\mathrm{SMD}=0.4795 \% \mathrm{CI}: 0.20$ to 0.74 . Unfortunately, selection bias (small-study effect) was present. For this outcome, the meta-regression showed that both presence of hypertension and the inclusion of nonbiased studies explained $28.3 \%$ of the heterogeneity among the studies. Simulation by the "trim and fill" method, which controlled for selection bias using random effects model, diluted the effect (SMD $=0.1795 \% \mathrm{CI}$ : -0.13 to 0.48 ). No effects by IPost on ST-segment resolution or on the majority of adverse clinical events were observed during follow up, except the incidence of congestive heart failure was found.

Conclusion: Evidence from this study suggests no cardioprotection from IPost, on surrogate and the majority of clinical end points. A possible beneficial effect on the incidence of congestive heart failure needs to be replicated by a large clinical trial.

Keywords: ischemic postconditioning, acute myocardial infarction, percutaneous coronary intervention

\section{Introduction}

Rapid, complete, and sustained reperfusion of the infarct-related coronary artery is a cornerstone in the treatment of acute ST-elevation myocardial infarction (STEMI). Primary percutaneous coronary intervention (PCI) is the preferred method of reperfusion if this can be performed within recommended time frames. ${ }^{1}$ However, in spite of revascularization and adjunctive medical treatment, mortality and morbidity after STEMI are still high. ${ }^{2}$ Paradoxically, reperfusion itself can enlarge the infarct size, by complex mechanisms collectively termed ischemia/reperfusion injury. ${ }^{3}$ 
In a previous study, different pharmacological principles that aimed to reduce ischemia/reperfusion injury failed to show effect. ${ }^{3}$ In 2003, Zhao and et all published experimental data demonstrating a considerable reduction of infarct size by a reperfusion procedure termed ischemic postconditioning (IPost), consisting of brief, repetitive cycles of reperfusion and reocclusion, followed by sustained reperfusion. ${ }^{4}$ An infarct-reducing effect of IPost was confirmed in several animal models, ${ }^{3}$ and subsequently, in small-sized "proof of concept" clinical studies, reduction of infarct size by IPost was reported. ${ }^{5-7}$ A previous review and meta-analysis ${ }^{8}$ indicated positive effects with IPost and that the outcomes of cardioprotection were affected by age, gender, comorbidities, and drugs, as well as by technical issues related to the determination of infarct size and revascularization procedure. More recently, larger, neutral clinical studies on IPost in STEMI have been published. ${ }^{9-12}$ Thus, data on the effects of IPost in STEMI treated by primary PCI have been diverging. We therefore conducted a systematic review and a critical appraisal, followed by a meta-analysis, to evaluate the effects of IPost on measures of infarct size and reperfusion, as well as on adverse clinical events during follow up.

\section{Methods}

\section{Literature search}

A qualified medical librarian was consulted at the Medical Library, Oslo University Hospital. We searched the Cochrane Central Register of controlled trials (1970-2014), Medline/ PubMed (1966-2014), and Embase (1980-2014). We used a combination of keywords, and details of the search procedure are shown in Figure 1. We used similar strategies to identify previously published meta-analyses and reviews. In addition, we manually searched conference proceedings and textbooks, screened reference lists of all papers, and contacted investigators, experts, and a translator. The last update for research was done on April 8, 2014. No limitation on language was considered.

\section{Trial selection}

We included randomized clinical trials (RCTs) on patients with STEMI and IPost intervention. Two reviewers independently evaluated the reports for eligibility. Disagreements were resolved by discussion. We had 21 studies included in our meta-analysis. ${ }^{5-7,9-26}$ The study of Roubille et al, ${ }^{27}$ in contrast with other researchers, investigated the effect of IPost in STEMI patients with reestablished coronary blood flow at admission. The design of their study differs

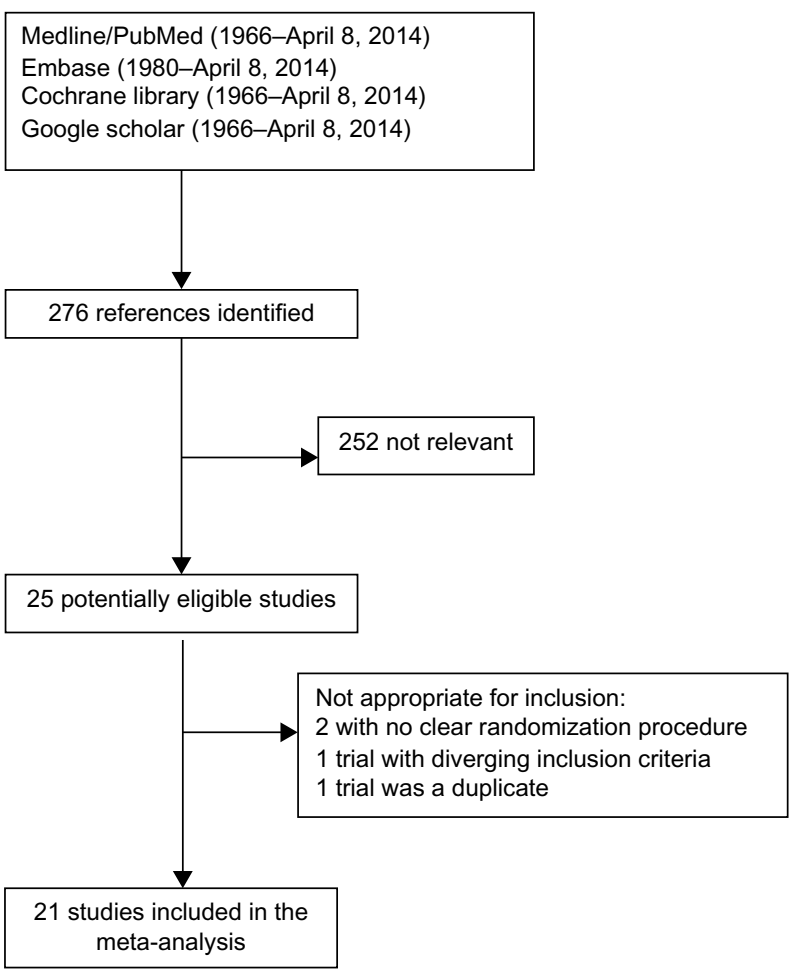

Figure I Flow chart of the literature searches for the systematic review of studies addressing the efficacy of IPost on infarct size.

Abbreviation: IPost, ischemic postconditioning.

fundamentally from that of the other studies of IPost, and we therefore did not include it in our review and analysis. Similarly, the study of Mewton et $\mathrm{al}^{28}$ was a duplicate of the study of Thuny et $\mathrm{al}^{22}$ and was not considered in our review and analysis.

\section{Quality assessment of the randomized trial: the component approach}

Two of the reviewers independently assessed randomization and concealment of treatment allocation (selection bias), blinding of investigators to outcome (detection bias), dropout rate (attrition bias), and adequacy of analyses according to "intention to treat". ${ }^{29}$ Concealment of allocation was considered adequate if the investigators responsible for patient selection were unable to suspect, before allocation, which treatment was next. Analyses were considered adequate if all recruited patients were analyzed in the group to which they were originally allocated, regardless of the treatment received (intention-to-treat principle). The problem of missing patients and dropouts in the analysis was evaluated according to Bell et al. ${ }^{30}$ Also, the beforehand power analysis of the trial was considered. This was done as advocated by the Cochrane Collaboration's tools for assessing risk of bias in randomized trials. ${ }^{29}$ 


\section{Data abstraction}

Data regarding publication status, trial design, patientrelated characteristics, treatment regimens, outcome methods, results, and funding were extracted in duplicate on a standardized form according to an a priori protocol. Investigators were contacted for additional data and corrections. Disagreements were resolved by discussion with a third reviewer and subsequent consensus. Patient-related variables were considered in the protocol and included mean age; presence of diabetes mellitus, hypertension, and dyslipidemia; current smoking habits; presence of anterior myocardial infarction; time from start of symptoms to PCI; and time to left ventricular ejection fraction (LVEF) evaluation after PCI.

\section{End points considered}

The primary end point was the infarct size, as estimated from cardiac magnetic resonance imaging (CMR); secondary outcomes were LVEF, as estimated by echocardiography or CMR at different times during follow up, and biomarkers of myocardial necrosis. Electrocardiographic (ECG) ST-segment resolution was evaluated as a marker of reperfusion. ${ }^{31}$ Adverse cardiac events during follow up (mortality, reinfarction, stent occlusion, and development of congestive heart failure $[\mathrm{CHF}]$ ) needed to be analyzed using the patient-years model, to control for variability in the duration of follow-up of the different trials.

\section{Quantitative data synthesis}

\section{Statistical pooling}

In order to calculate the "pooled" effect of intervention, the total standardized mean difference (SMD), with 95\% confidence interval (CI), was estimated for fixed and random effects models (Cohen's method). If the value of zero is not included in the $95 \% \mathrm{CI}$, then the SMD is statistically significant at the $5 \%$ level $(P<0.05)$. Cohen's rule of thumb for interpretation of the SMD statistics is: a value of 0.2 indicates a small effect, of 0.5 indicates a medium effect, and of 0.8 and over indicates a large effect. As the homogeneity hypothesis appeared to be irrelevant, we worked with a random effects model. The estimation was done according to the DerSimonian and Laird method, ${ }^{32}$ and fitted for a pooled analysis. For each model, a test for overall effect was done. Because of the heterogeneity of our studies, the random effects model was more appropriate. Using this model, the estimate of the pooled effect measure and its $\mathrm{CI}$ incorporates the additional variability due to interstudy variance $\left(\tau^{2}\right)$.

\section{Sources of heterogeneity, evaluation, and quantification}

Statistical heterogeneity among studies was assessed with Cochran's $Q$ test. The magnitude of heterogeneity was evaluated by the $I^{2}$ statistics, which describes the proportion of total variation due to heterogeneity rather than chance. $I^{2}$-values of $25 \%, 50 \%$, and $75 \%$ indicate low, moderate, and high heterogeneity, respectively. ${ }^{33}$ In order to investigate possible sources of heterogeneity, two different methods were used: subgroup analyses and meta-regression. We stratified our data according to the following study characteristics: concealment of randomization, blinding of the end point assessors on allocated treatment, analyses according to intention-to-treat strategy, and presence of a priori power estimation for the study. The test for between-group heterogeneity is an issue of current debate as it is strictly valid when using the fixed effect inverse variance method, and this test is likely to be invalid when considerable heterogeneity is observed in one or more subgroups. After doing the subgroup analyses, we ran a random effects meta-regression analysis, where the outcome variable was the observed SMD from every study, indicating treatment effect, and study-level and patient-level characteristics (covariates). This random effects regression model was done to enable exploration of the source of heterogeneity in the association between intervention and outcomes. The choice of covariates was based on literature reviews and motivated by biological and clinical hypotheses, supported by evidence from sources other than the included studies. A source of heterogeneity was considered as important if the covariate decreased the between-study variance. The estimate of $\tau^{2}$ in the presence of a covariate, in comparison to that when the covariate is omitted, allows the proportion of the heterogeneity variance explained by the covariate to be calculated. ${ }^{34}$

\section{Publication bias or small-study effect}

Publication bias is known to occur in meta-analyses as studies with results that are significant, interesting, from large wellfunded studies, or from studies of higher quality are more likely to be submitted and published. A meta-analysis based on a literature search will thus include such studies differentially, and the resulting bias may invalidate the conclusions. In order to assess potential publication bias, or "small-study effect", we used the funnel plot, which provides a good visual evaluation of sampling bias. Funnel plot asymmetry raises the possibility of bias but is not a proof of bias. It is important to note that asymmetry (unless produced by chance alone) will always lead to a questioning of the interpretation of the 
overall effect when studies are combined in a meta-analysis. Sterne et $\mathrm{al}^{35}$ have suggested that the funnel plot should be seen as a generic means of examining small-study effects. Small-study effect is the tendency for the smaller studies in a meta-analysis to show larger treatment effects. A test of asymmetry (Egger's test of the intercept), applied on the funnel plot, was performed. ${ }^{36}$ According to this, if the intercept on the y-axis is null or if its $95 \% \mathrm{CI}$ crosses the zero line, there is no publication bias or small-study effect. It is a good practice to avoid evaluating the publication bias only according to visual judgment. Nowadays Egger's test is mostly seen as a test of small-study effects.

\section{Detection and correction for publication bias using the trim and fill simulation method}

Publication bias is a serious issue as it may lead to biased estimates that appear to be precise. A popular method for detecting and adjusting bias is the "trim and fill" method. ${ }^{37}$ This method is suitable also for continuous outcome effects. ${ }^{38}$ Peters et $\mathrm{al}^{39}$ concluded that in the presence of publication bias, the trim and fill method can help reduce bias in pooled estimates. Even though its performance is not ideal, this method is used as a sensitivity analysis for assessing the potential impact of missing studies. This method begins by estimating the number of asymmetric trials on the right side of the funnel plot. These can broadly be thought of as trials that have no left-side counterpart. These trials are then removed or "trimmed" from the funnel plot, leaving a symmetric remainder from which the true center of the funnel is estimated. The trimmed trials are then replaced and their missing counterparts imputed or "filled". This, in turn, allows an adjusted overall CI to be calculated. A test of the presence of bias could be derived from this method, based on the estimated number of missing trials. The estimated effect of the missing trials provides an indication of whether the imputed missing studies affect the overall result of the meta-analysis.

A simulation study, based on 1,000 replications (reflecting the results of our meta-analysis for the end point incidence of $\mathrm{CHF}$ ), was done to predict the sample size of a future RCT to be performed..$^{40,41}$ This analysis is interesting if we want to assess the impact of a potential new trial on the overall evidence from our meta-analysis. Also, it permits us to assess the robustness of the current meta-analysis with respect to the inclusion of future data.

The test of funnel plot asymmetry and meta-regression analysis can only be used when at least ten studies are included in the meta-analysis, because of power limitations.
Under the fixed effect model, the power of the meta-analysis is driven by the number of subjects accumulated across studies and the effect size we want to highlight. Under the random effects model, power depends, not only on the total number of subjects, but also, on the number of studies, the effect size, and the variance between studies (heterogeneity). ${ }^{34,42}$

All statistical analyses were performed with STATA 13.0 (StataCorp LP, College Station, TX, USA).${ }^{43}$ We followed the Quorum guidelines for meta-analyses and systematic reviews of randomized trials in the present report. ${ }^{44}$

\section{Results}

\section{Trial flow}

After identifying 276 references, 252 were excluded due to irrelevant content and duplicate publications, leaving 25 potentially eligible studies. Two were not appropriate for inclusion because of unclear randomization procedure, one had diverging inclusion criteria, and one was a duplicate report of another study. Finally, 21 studies were included in our systematic review (Figure 1).

\section{Study characteristics}

The study characteristics are presented in Tables 1 and 2. Regarding patient-related variables, the mean age was 59 years (standard deviation [SD] =2.2 years), 77\% were males, and $49 \%$ were smokers, $44 \%$ had hypertension, $48 \%$ had dyslipidemia, 20\% had diabetes mellitus, and 51\% had anterior myocardial infarction. On average, time from symptom onset to PCI was 246 minutes ( $\mathrm{SD}=63$ minutes) and median time from PCI to assessment of outcome was 60 days (interquartile range 7, 180 days). As for the study-level characteristics, randomization and adequate concealment were present in $67 \%$ of the trials, blinding of the end point assessor to treatment allocation was present in $81 \%$, intention-to-treat strategy was followed in $76 \%$, and a priori power estimation was presented in $43 \%$. Report of dropouts was present in $43 \%$ of the trials. The nonbiased and powered studies represented $43 \%$ of the included studies.

\section{Quantitative data synthesis}

Efficacy of IPost on infarct size estimated by CMR

The results are summarized in Figure 2. Our pooled analysis included eight trials showing a pooled effect of $\mathrm{SMD}=-0.062$ (95\% CI: -0.34 to $0.21, P=0.625)$ with a moderate heterogeneity $\left(I^{2}=68 \%\right)$, indicating no effect of IPost on infarct size, as estimated by CMR. The majority of these trials were of good quality and as the number of trials was 
Table I Clinical profile of the 21 randomized trials included in the meta-analysis

\begin{tabular}{|c|c|c|c|c|}
\hline Study & Protocol $^{a}$ & $\begin{array}{l}\text { IPost/ } \\
\text { control, } \\
\text { N/N }\end{array}$ & $\begin{array}{l}\text { Symptom } \\
\text { set, hrs }\end{array}$ & End points \\
\hline Staat et $\mathrm{al}^{5}$ & $60 s \times 4$ & $14 / 16$ & $<6$ & CK \\
\hline Laskey ${ }^{13}$ & $90 s \times 2$ & $10 / 7$ & $<12$ & CK \\
\hline Ma et $\mathrm{al}^{14}$ & $30 s \times 3$ & $47 / 47$ & $\leq 12$ & CK, LVEF \\
\hline Yang et $\mathrm{al}^{6}$ & $30 s \times 3$ & $23 / 18$ & $>12$ & CK, LVEF, STR \\
\hline Thibault et al ${ }^{7}$ & $60 s \times 4$ & $|7 / 2|$ & $<6$ & LVEF, CK, TN \\
\hline Laskey et al ${ }^{15}$ & $90 s \times 2$ & $12 / 12$ & $\leq 6$ & CK, LVEF \\
\hline $\begin{array}{l}\text { Lønborg } \\
\text { et } \mathrm{al}^{45}\end{array}$ & $30 s \times 4$ & $59 / 59$ & $<12$ & $\begin{array}{l}\text { MISIZE, TN, } \\
\text { LVEF, ACE, STR }\end{array}$ \\
\hline $\begin{array}{l}\text { Sörensson } \\
\text { et al }\end{array}$ & $60 s \times 4$ & $38 / 38$ & $<6$ & $\begin{array}{l}\text { MISIZE, LVEF, } \\
\text { CK }\end{array}$ \\
\hline Xue et al ${ }^{17}$ & $60 s \times 4$ & $23 / 20$ & $\leq 12$ & CK, LVEF, STR \\
\hline Lin et $\mathrm{al}^{18}$ & $60 s \times 3$ & $58 / 63$ & & LVEF \\
\hline Garcia et al ${ }^{19}$ & $30 s \times 4$ & $22 / 21$ & $<12$ & LVEF, ACE, CK \\
\hline Liu et $\mathrm{al}^{20}$ & $30 s \times 3$ & $30 / 34$ & $<12$ & CK-MB, LVEF \\
\hline Freixa et al" & $60 s \times 4$ & $30 / 31$ & $<12$ & $\begin{array}{l}\text { MISIZE, LVEF, } \\
\text { CK, CK-MB,TN }\end{array}$ \\
\hline Zhao et $\mathrm{al}^{21}$ & $60 s \times 4$ & $32 / 30$ & $<12$ & TN, LVEF \\
\hline $\begin{array}{l}\text { Tarantini } \\
\text { et al }{ }^{10}\end{array}$ & $60 s \times 4$ & $39 / 39$ & $<6$ & $\begin{array}{l}\text { MISIZE, LVEF, } \\
\text { TN }\end{array}$ \\
\hline Thuny et $\mathrm{al}^{22}$ & $60 s \times 4$ & $25 / 25$ & $\leq 12$ & $\begin{array}{l}\text { MISIZE, LVEF, } \\
\text { CK }\end{array}$ \\
\hline $\begin{array}{l}\text { Elżbieciak } \\
\text { et } \mathrm{al}^{23}\end{array}$ & $60 s \times 4$ & $|8 / 2|$ & $\leq 12$ & $\begin{array}{l}\text { MISIZE, LVEF, } \\
\text { ACE }\end{array}$ \\
\hline Dwyer et $\mathrm{a}^{24}$ & $30 s \times 4$ & $50 / 52$ & $\leq 6$ & $\begin{array}{l}\text { MSIZE, LVEF, } \\
\text { CK }\end{array}$ \\
\hline Hahn et $\mathrm{a}^{25}$ & $60 s \times 4$ & $350 / 350$ & $<12$ & STR, ACE \\
\hline Dong et $\mathrm{a}^{26}$ & $30 s \times 3$ & $32 / 30$ & $<12$ & TN, LVEF, STR \\
\hline $\begin{array}{l}\text { Limalanathan } \\
\text { et al' }\end{array}$ & $60 s \times 4$ & $136 / 136$ & $\leq 6$ & $\begin{array}{l}\text { MISIZE, LVEF, } \\
\text { TN, ACE }\end{array}$ \\
\hline
\end{tabular}

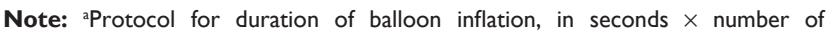
inflations.

Abbreviations: $A C E$, adverse clinical events; CK, creatine kinase; CK-MB, creatine kinase-MB fraction; IPost, ischemic postconditioning; LVEF, left ventricular ejection fraction; MISIZE, anatomic infarct size estimated by cardiac magnetic resonance imaging; STR, ST-segment resolution; TN, troponin.

lower than ten, no meta-regression analysis was performed over the patient- and study-level variables.

\section{Effect of IPost on biomarkers of myocardial necrosis}

The pooled estimate including 18 studies, using the random effects model, showed that the release of markers of myocardial necrosis (creatine kinase $[\mathrm{CK}]$, creatine kinase-MB fraction [CK-MB], and troponin) after PCI was significantly reduced by IPost (SMD $=-0.58,95 \% \mathrm{CI}:-0.96$ to -0.19 ), with an $I^{2}$ of $90.1 \%$, indicating that $90.1 \%$ of the variation in the SMD was attributable to heterogeneity (Figure 3). There was no indication of publication bias as the funnel plot was satisfactory and Egger's test rejected the null hypothesis of small-study effect.
Analyses specific for each biomarker as outcome showed that for the more cardio-specific biomarkers, troponin and CK-MB, no effect of IPost was found (SMD $=-0.27,95 \%$ CI: -0.60 to $0.07, P=0.120$ and $\mathrm{SMD}=-0.54,95 \% \mathrm{CI}:-1.66$ to $0.58, P=0.349$, respectively). Regarding the less cardiospecific marker, CK, IPost significantly reduced the level (SMD $=-0.84,95 \% \mathrm{CI}:-1.37$ to $-0.31, P=0.003$ ).

An investigation to identify the main source of heterogeneity was done by subgroup analysis of major parameters of critical appraisal (Table 2) - randomization and concealment, blinding of end point assessor, intention-to-treat strategy, and account for the dropouts that could not be quantified correctly, as some studies ignored this. As well, the problem of differential drop-out rate was considered carefully for every publication. The components of internal validity were correlated as the studies of good quality were conducted according to evidence-based criteria. We generated a variable called "powered and nonbiased studies", which was defined as "yes" if the study was satisfactory with regard to the aforementioned parameters, otherwise as "no". Our results are summarized in Table 3. The nonbiased and powered studies showed no effect of IPost on biomarkers of myocardial necrosis, while the trials with deficient methodology showed a beneficial effect.

\section{Effect of IPost on LVEF}

The measurement of LVEF was done either early, before discharge from the hospital, or during follow up. Unfortunately, no study presented change in LVEF. Thus, the pooled analysis was done on group differences in LVEF measured either in the acute stage or after follow up, rather than on between-group changes from baseline.

In the first meta-analysis, 18 trials were considered. A pooled effect showed a moderate improvement of LVEF ( $\mathrm{SMD}=0.47,95 \% \mathrm{CI}: 0.20$ to 0.74 ), with substantial heterogeneity $\left(I^{2}=80.8 \%\right)$, using the random effects model. On the other hand, the funnel plot visually showed the possibility of bias or small-study effect. This was confirmed by the Egger's test, which had $P$-value of 0.01 , accepting the null hypothesis of small-study effect (Figure 4).

The presence of small-study effect led us to an investigation of its origin. It is possible that differences between smaller and larger trials are accounted for by trial characteristics. The most important characteristic for the stratification analyses was the study-level variable "powered and non-biased studies". The stratified, pooled meta-analysis (Table 4) showed that the "powered and nonbiased studies" (eight studies) demonstrated no effect of IPost on LVEF 
Table 2 Study-level characteristics ${ }^{\mathrm{a}}$ of included trials

\begin{tabular}{|c|c|c|c|c|c|c|}
\hline Study & $\begin{array}{l}\text { Concealment of } \\
\text { randomization }\end{array}$ & $\begin{array}{l}\text { Blinding of assessor to } \\
\text { outcome treatment group }\end{array}$ & $\begin{array}{l}\text { Intention to } \\
\text { treat strategy }\end{array}$ & $\begin{array}{l}\text { A priori power } \\
\text { estimation }\end{array}$ & $\begin{array}{l}\text { Composite } \\
\text { conditions }^{b}\end{array}$ & $\begin{array}{l}\text { Dropouts during } \\
\text { follow up }\end{array}$ \\
\hline Staat et $\mathrm{al}^{5}$ & 0 & I & 0 & 0 & 0 & 0 \\
\hline Laskey ${ }^{13}$ & 0 & 0 & I & 0 & 0 & 0 \\
\hline Ma et $\mathrm{al}^{14}$ & 0 & I & 0 & 0 & 0 & 0 \\
\hline Yang et $\mathrm{al}^{6}$ & 0 & 0 & 0 & 0 & 0 & 0 \\
\hline Thibault et $\mathrm{al}^{7}$ & I & I & I & I & I & 0 \\
\hline Laskey et al' ${ }^{15}$ & I & I & I & I & I & 0 \\
\hline Lønborg et al ${ }^{45}$ & I & 1 & I & I & I & 1 \\
\hline Sörensson et $\mathrm{al}^{9}$ & I & I & I & I & I & I \\
\hline Xue et $\mathrm{al}^{17}$ & 0 & I & 0 & 0 & 0 & 0 \\
\hline Lin et $\mathrm{al}^{18}$ & I & 1 & I & 0 & 0 & 0 \\
\hline Garcia et al ${ }^{19}$ & 0 & 0 & 0 & 0 & 0 & 0 \\
\hline Liu et $\mathrm{al}^{20}$ & I & 0 & I & 0 & 0 & 0 \\
\hline Freixa et al" & I & I & I & I & I & I \\
\hline Zhao et $\mathrm{al}^{21}$ & 0 & I & I & 0 & 0 & 0 \\
\hline Tarantini et al ${ }^{10}$ & I & I & I & I & I & I \\
\hline Thuny et $\mathrm{al}^{22}$ & I & I & I & 0 & 0 & I \\
\hline Elżbieciak et a ${ }^{23}$ & I & I & I & 0 & 0 & 0 \\
\hline Dwyer et $\mathrm{a}^{24}$ & I & I & I & I & I & I \\
\hline Hahn et $\mathrm{al}^{25}$ & I & I & I & I & I & I \\
\hline Dong et $\mathrm{a}^{26}$ & I & I & I & 0 & 0 & I \\
\hline Limalanathan et $\mathrm{al}^{12}$ & I & I & I & I & I & 1 \\
\hline
\end{tabular}

Notes: ${ }^{\mathrm{a}} \mathrm{I}=$ presence of the condition, $0=$ absence of the condition or unclear; ${ }^{\mathrm{b}}$ composite variable that has the value of $\mathrm{I}$ when the following conditions are respected or present simultaneously: concealment of randomization, end point assessor blinded to treatment group, intention-to-treat strategy, and a priori power estimation (nonbiased and powered studies).

(SMD $=0.17,95 \% \mathrm{CI}:-0.02$ to $0.36, P=0.082$ ), while for studies with nonpresence of this condition (ten studies), a significant effect of IPost on LVEF was found (SMD $=0.71$, 95\% CI: 0.23 to $1.19, P=0.004)$.

We extended the analyses with a meta-regression model, taking into account patient-related variables (mean age, frequency of diabetes mellitus, presence of hypertension and dyslipidemia, presence of anterior myocardial infarction, current smoking habits, time from symptom onset to PCI, and time of evaluation of LVEF after PCI). Two important patientrelated variables were identified - presence of hypertension and smoking habits. Both of these were associated with

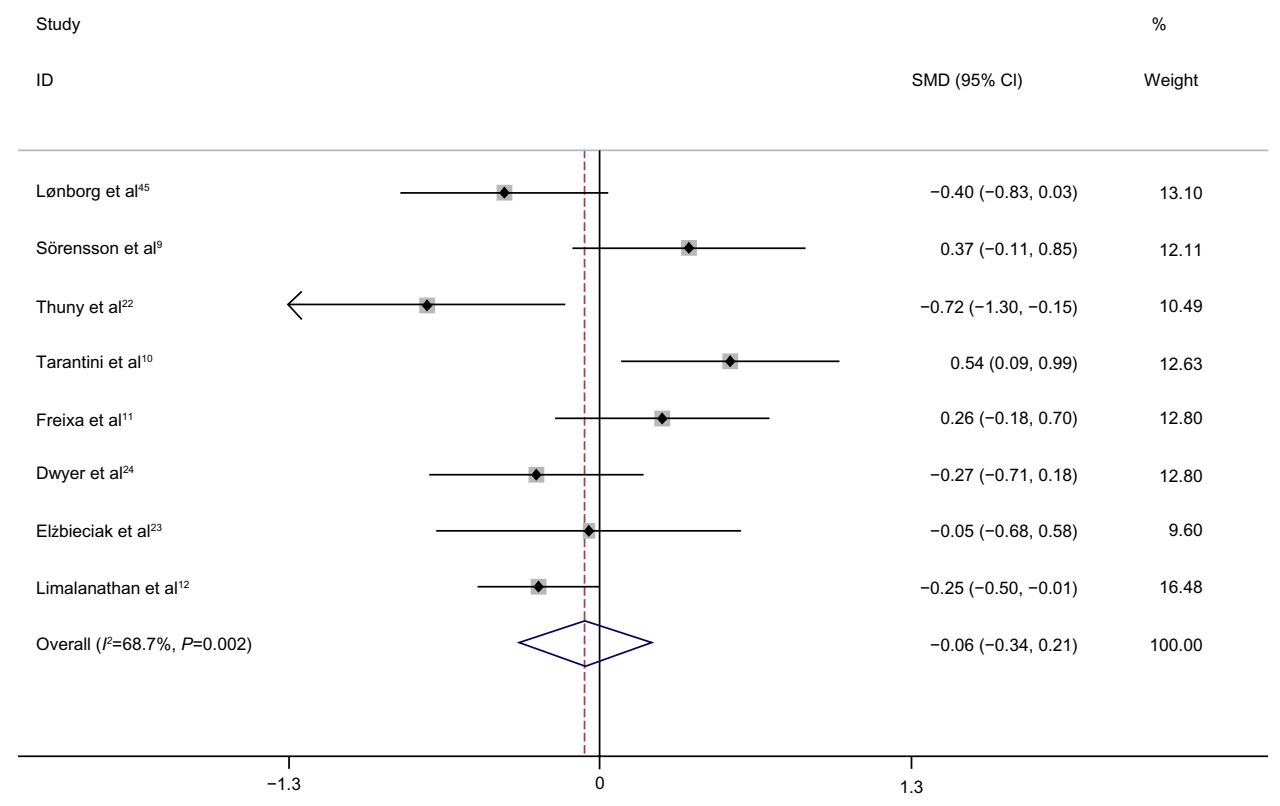

Figure 2 Forest plot of anatomic infarct size as estimated by CMR, expressed as SMD.

Note: Weights are from random effects analysis.

Abbreviations: $\mathrm{Cl}$, confidence interval; CMR, cardiac magnetic resonance imaging; SMD, standardized mean difference. 


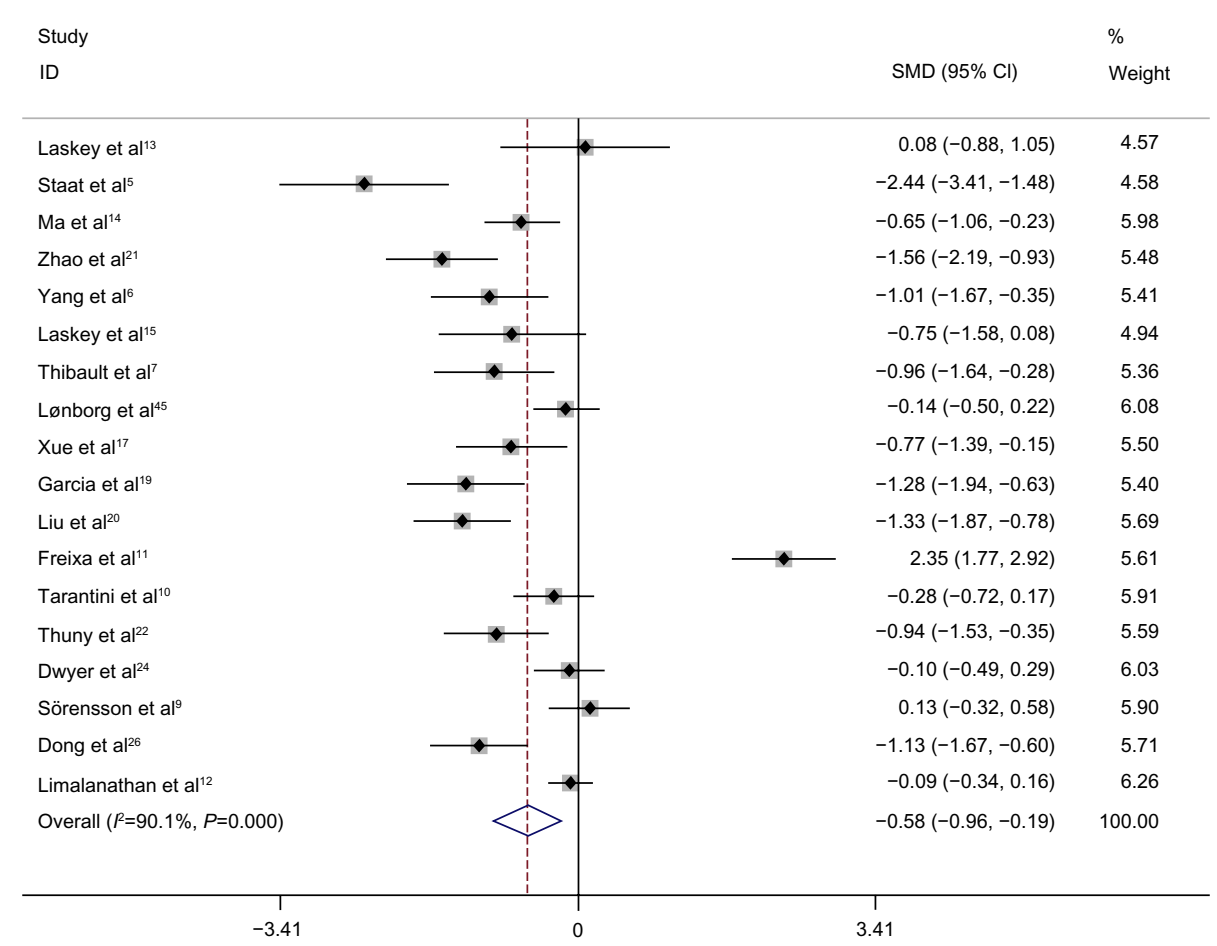

Figure 3 Forest plot for biomarkers of myocardial necrosis, expressed as standardized mean difference SMD.

Note: Weights are from random effects analysis.

Abbreviations: $\mathrm{Cl}$, confidence interval; SMD, standardized mean difference.

reduced effect of IPost and explained $17.4 \%$ and $27.4 \%$ of heterogeneity, respectively (Figure 5A and B). However, these two covariates were moderately correlated in our study $(R=0.64)$. Finally, a multivariate meta-regression was performed, which considered study-level and patient-level variables together. The multivariate method identified the presence of hypertension and "powered and nonbiased studies" as major reasons of heterogeneity, with $28.3 \%$ of heterogeneity accounted for by these two variables together (Table 5).

Table 3 Subgroup analysis performed with study-level characteristics considered as potential sources of heterogeneity for the outcome biomarkers of myocardial necrosis

\begin{tabular}{|c|c|c|c|c|c|}
\hline Subdivision & $\mathbf{N}$ & SMD (95\% Cl) & $\begin{array}{l}\text { SMD }=0 \\
Z\end{array}$ & $P$-value & $p^{2 a} \%$ \\
\hline All studies & 18 & $-0.58(-0.96,-0.19)$ & 2.94 & 0.003 & 90.1 \\
\hline \multicolumn{6}{|c|}{ Concealment of randomization } \\
\hline Yes & 11 & $-0.28(-0.76,0.19)$ & 1.16 & 0.246 & 91.6 \\
\hline Inadequate/unclear & 7 & $-0.57(-0.96,-0.19)$ & 4.53 & 0.0001 & 70.7 \\
\hline \multicolumn{6}{|c|}{ Outcome assessor blinded to treatment group } \\
\hline Yes & 14 & $-0.48(-0.92,0.05)$ & 2.18 & 0.029 & 91.2 \\
\hline Inadequate/unclear & 4 & $-0.99(-1.50,-0.47)$ & 3.77 & 0.0001 & 55.2 \\
\hline \multicolumn{6}{|c|}{ Intention-to-treat strategy } \\
\hline Yes & 13 & $-0.46(-0.92,0.01)$ & 1.93 & 0.053 & 91.8 \\
\hline No/unclear & 5 & $-0.91(-1.51,-0.32)$ & 3.01 & 0.003 & 73.6 \\
\hline \multicolumn{6}{|c|}{ A priori power estimation } \\
\hline Yes & 8 & $0.03(-0.48,0.55)$ & 0.12 & 0.902 & 91.0 \\
\hline No/unclear & 10 & $-0.58(-0.96,-0.19)$ & 6.90 & 0.0001 & 63.4 \\
\hline \multicolumn{6}{|c|}{ Dropouts during follow up } \\
\hline Yes & 8 & $-0.03(-0.57,0.5 \mathrm{I})$ & 0.11 & 0.910 & 92.5 \\
\hline No/unclear & 10 & $-1.06(-1.38,-0.73)$ & 6.31 & 0.0001 & 59.5 \\
\hline \multicolumn{6}{|c|}{ Nonbiased and powered studies } \\
\hline Yes & 8 & $0.03(-0.48,0.55)$ & 0.12 & 0.902 & 91.0 \\
\hline No/unclear & 10 & $-1.08(-1.39,-0.77)$ & 6.90 & 0.0001 & 59.0 \\
\hline
\end{tabular}

Note: ${ }^{a}{ }^{2}=$ the variation in SMD due to heterogeneity.

Abbreviations: $\mathrm{Cl}$, confidence interval; SMD, standardized mean difference. 


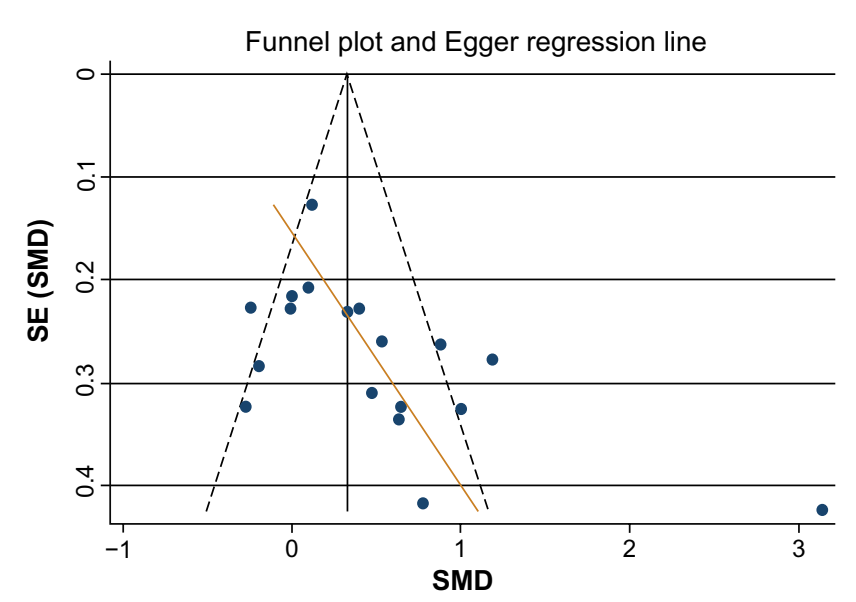

Figure 4 Funnel plot of the effect of IPost on left ventricular ejection fraction. Abbreviations: IPost, ischemic postconditioning; SMD, standardized mean difference; SE, standard error.

To control for selection bias, we considered the trim and fill simulation method, to look for missing studies based on the fixed effect model, and looked for missing studies only to the "left side" of the mean effect. Using these parameters, the method suggested five studies as missing. Under the random effects model, the point estimate and 95\% CI for the combined studies, as shown earlier, was SMD $=0.47,95 \%$ CI: 0.20 to $0.74, P=0.001$. Using the trim and fill method, the imputed point estimate was altered, SMD $=0.17,95 \%$ CI: -0.13 to $0.48, P=0.270$. This indicates a change in magnitude and significance of the pooled effect after correction for publication bias or small-study effect. As well, Egger's test no longer showed evidence of publication bias. These results are in concordance with lack of effect of IPost on LVEF, as highlighted in the subgroup of "powered and nonbiased studies".

\section{Effect of IPost on reperfusion, assessed by ECG ST-segment resolution}

ST-segment resolution after PCI has been considered a marker of myocardial reperfusion (Table 6). We analyzed six trials, three of which considered ST-resolution $>70 \%$ at 30,90 , or 120 minutes after PCI, while the three remaining trials considered ST-resolution $>50 \%$ at 60 or 120 minutes to indicate successful reperfusion. The variation of the pooled estimate attributable to heterogeneity was moderate $(I=31.3 \%)$. The pooled estimate effect (rate ratio) was $\mathrm{RR}=1.08,95 \% \mathrm{CI}$ : 0.95 to $1.21, P=0.322$, indicating no effect of IPost on STsegment resolution.

\section{Effect of IPost on adverse cardiac events during follow up, using the patient-years model}

The results of six trials are summarized in Table 7, using the patient-years model to control for variability in the duration of follow up. We included the study by Lønborg et al, with the longest follow-up time. ${ }^{45}$ Analysis showed no effect of IPost

Table 4 Subgroup analysis performed according to study-level characteristics considered as potential sources of heterogeneity for the outcome LVEF

\begin{tabular}{|c|c|c|c|c|c|}
\hline Subdivision & $\mathbf{N}$ & SMD (95\% Cl) & $\begin{array}{l}\text { SMD =0 } \\
Z\end{array}$ & $P$-value & $P^{2 \mathrm{a}} \%$ \\
\hline All studies & 18 & $0.47(0.20,0.74)$ & 3.46 & 0.001 & 80.8 \\
\hline \multicolumn{6}{|c|}{ Concealment of randomization } \\
\hline Yes & 13 & $0.46(0.11,0.81)$ & 2.59 & 0.010 & 85.1 \\
\hline Inadequate/unclear & 5 & $0.49(0.19,0.80)$ & 3.18 & 0.001 & 36,1 \\
\hline \multicolumn{6}{|c|}{ Outcome assessor blinded to treatment group } \\
\hline Yes & 15 & $0.44(0.13,0.74)$ & 2.83 & 0.005 & 83.0 \\
\hline Inadequate/unclear & 3 & $0.47(0.20,0.74)$ & 4.07 & 0.0001 & 00.0 \\
\hline \multicolumn{6}{|c|}{ Intention-to-treat strategy } \\
\hline Yes & 14 & $0.46(0.14,0.79)$ & 2.79 & 0.005 & 84.0 \\
\hline No/unclear & 4 & $0.50(0.10,0.90)$ & 2.47 & 0.014 & 51.2 \\
\hline \multicolumn{6}{|c|}{ A priori power estimation } \\
\hline Yes & 7 & $0.15(-0.06,0.36)$ & 1.38 & 0.168 & 37.5 \\
\hline No/unclear & 11 & $0.67(0.24,1.10)$ & 3.08 & 0.002 & 84.8 \\
\hline \multicolumn{6}{|l|}{ Dropouts } \\
\hline Yes & 8 & $0.03(-0.57,0.5 \mathrm{I})$ & 0.11 & 0.910 & 92.5 \\
\hline No/unclear & 10 & $-1.06(-1.38,-0.73)$ & 6.31 & 0.0001 & 59.5 \\
\hline \multicolumn{6}{|c|}{ Nonbiased and powered studies } \\
\hline Yes & 8 & $0.17(-0.02,0.36)$ & 1.74 & 0.082 & 32.0 \\
\hline No & 10 & $0.71(0.23,1.19)$ & 2.91 & 0.004 & 86.1 \\
\hline
\end{tabular}

Note: ${ }^{a}{ }^{2}=$ the variation in SMD due to heterogeneity.

Abbreviations: LVEF, left ventricular ejection fraction; SMD, standardized mean difference; $\mathrm{Cl}$, confidence interval. 

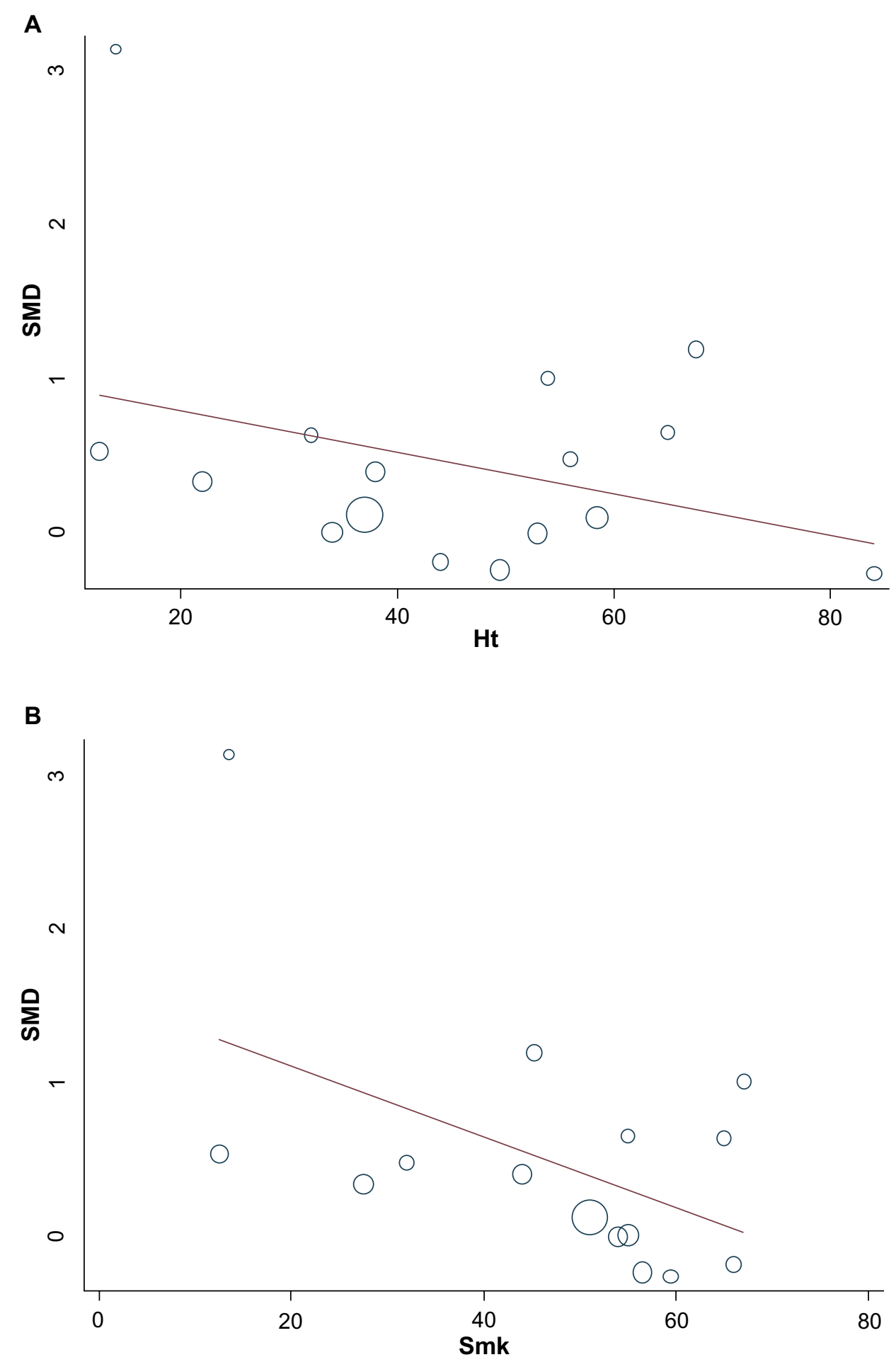

Figure 5 Meta-regression of left ventricular ejection fraction and (A) proportion of hypertension (\%) and (B) proportion of smoking (\%).

Abbreviations: $\mathrm{Ht}$, hypertension; SMD, standardized mean difference; Smk, smoking.

on mortality $(\mathrm{RR}=1.49,95 \% \mathrm{CI}: 0.84-2.64, P=0.167)$, the incidence of reinfarction $(\mathrm{RR}=1.50,95 \% \mathrm{CI}: 0.84$ to 2.67 , $P=0.171)$ or the incidence of stent thrombosis $(\mathrm{RR}=1.17$, 95\% CI: $0.50-2.74, P=0.708)$, or on the frequency of the composite end point of major adverse cardiac events (RR $=1.16,95 \% \mathrm{CI}: 0.71-1.89, P=0.564)$. A significant beneficial effect of IPost was found on the incidence of new onset CHF ( $\mathrm{RR}=0.43,95 \% \mathrm{CI}: 0.23$ to $0.77, P=0.005$ ), with no heterogeneity during the follow-up period $\left(I^{2}=0 \%\right)$. CHF was defined in the majority of studies ${ }^{12,19,26}$ as a "hard" clinical end point - rehospitalization for CHF. Hahn et $\mathrm{al}^{25}$ considered $\mathrm{CHF}$ patients in need of intensive care, whereas 
Table 5 Estimate of the random effects regression model' between the SMD of left ventricular ejection fraction and the different study-level and patient-level variables, in a multivariate analysis using the 16 randomized clinical trials

\begin{tabular}{llllllll}
\hline Covariates & Level & $\beta$-coefficient & Std err $(\beta)$ & $\mathbf{Z}$ & $\boldsymbol{P}$-value & $\tau^{2 \mathrm{a}}$ & Heterogeneity $^{\mathrm{b}}$ \\
\hline $\begin{array}{l}\text { Presence of } \\
\text { hypertension }\end{array}$ & Yes/no & -0.0201 & 0.0094 & -2.12 & 0.054 & 0.3291 & $28.3 \%$ \\
$\begin{array}{l}\text { Nonbiased and } \\
\text { powered studies }\end{array}$ & Yes/no & -0.7772 & 0.3553 & -2.19 & 0.048 & & \\
\hline
\end{tabular}

Notes: ${ }^{\mathrm{a}} \tau^{2}=$ between-study variance, $\tau^{2}=0.4589$ model without covariates; ${ }^{\mathrm{b}}$ the heterogeneity accounted by the covariate included in the random effect meta-regression was $\tau^{2}-\tau^{2} / \tau^{2} \times 100$, where $\tau_{0}^{2}=$ without the covariates and $\tau_{\mathrm{m}}^{2}=$ including the two covariates.

Abbreviations: $\mathrm{Cl}$, confidence interval; SMD, standardized mean difference; Std err, standard error of the regression coefficient.

Lønborg et $\mathrm{a}^{45}$ used a softer CHF definition, ie, patients with New York Heart Association (NYHA) class 2-4 at 15 months follow up. This peculiarity for the outcome CHF led us to simulate a new two-arm trial using the results of our metaanalysis. This trial had 100 patients in each arm. For this calculation, we considered the pooled fixed effect model from our meta-analyses $(\mathrm{RR}=0.43)$. This gave $\ln R R=\ln (0.43)$ equaling -0.8439 , and the estimated variance of $\ln R R$ equaling 0.1019 in the equation

$$
\mathrm{V}=\left(\frac{\operatorname{lnRR} / \mathrm{RR}_{\mathrm{L}}}{1.96}\right)^{2}
$$

where $R R$ was the effect size and $R_{L}$ was the lower $95 \%$ $\mathrm{CI}$ of the effect size. Another simulation, with 1,000 replications, was done and gave us a power estimate of $80 \%$ with $95 \%$ CI: 70.8 to 87.3 , indicating that the $P$-value was below 0.01 in 80 of 100 iterations. Finally, we generated a power curve for the future meta-analysis. Our results are summarized in Figure 6, indicating that a future trial with a total sample size of 360 patients with 180 in each arm will ensure a meta-analysis with acceptable power close to $84 \%$, with $95 \%$ CI: 81.6 to 86.3 . The result of our simulation implies that when more data are available, a new meta-analysis could provide a conclusive answer

Table 6 A meta-analysis of six trials for the outcome ST-segment resolution as a marker of reperfusion

\begin{tabular}{|c|c|c|c|c|}
\hline Study considered & $\begin{array}{l}\text { Rate } \\
\text { ratio }\end{array}$ & $95 \% \mathrm{Cl}$ & $\begin{array}{l}\text { Time after } \\
\text { PCI }\end{array}$ & $\begin{array}{l}\text { ST } \\
\text { resolution }\end{array}$ \\
\hline Yang et al ${ }^{6}$ & 1.12 & $0.53-2.35$ & 120 minutes & $>70 \%$ \\
\hline Lønborg et al ${ }^{45}$ & 1.29 & $0.96-1.75$ & 90 minutes & $>70 \%$ \\
\hline Xue et al ${ }^{17}$ & 1.84 & $1.09-3.09$ & 120 minutes & $>50 \%$ \\
\hline Hahn et $\mathrm{al}^{25}$ & 0.98 & $0.82-1.17$ & 30 minutes & $>70 \%$ \\
\hline Dong et $\mathrm{al}^{26}$ & 1.28 & $1.01-1.61$ & 120 minutes & $>50 \%$ \\
\hline Limalanathan et al' & 1.02 & $0.93-1.10$ & 60 minutes & $>50 \%$ \\
\hline Pooled effect estimate & $\mathrm{I} .08$ & $0.95-1.21$ & & \\
\hline
\end{tabular}

Note: ${ }^{\text {a }}$ ariation in pooled estimate attributable to heterogeneity is $31.3 \%$. Abbreviations: $\mathrm{Cl}$, confidence interval; $\mathrm{PCl}$, percutaneous coronary intervention. regarding the effect of IPost on the incidence of CHF during follow up.

\section{Discussion}

Our meta-analysis of trials comparing IPost with usual care revealed a high degree of heterogeneity among trials for all the outcomes, except adverse cardiac events, during follow up. Also, publication bias or small-size effect was found to influence the outcome LVEF. Meta-regression analyses indicated that heterogeneity could be explained by patient-related variables and study-level characteristics. For studies reporting infarct size determined by CMR, probably the most robust and biologically relevant of the surrogate end points (SEPs) in small- and medium-sized STEMI trials,${ }^{46}$ there was no effect of IPost on infarct size. Also, regarding the SEPs LVEF, ST-segment resolution, and the majority of clinical end points, there was no effect of IPost, except for a possible beneficial effect on the incidence of CHF after the intervention. It has been claimed that direct stenting, as opposed to stenting after predilation, is important for cardioprotection by IPost. ${ }^{47}$ However positive effects of IPost have also been reported in studies using conventional stenting. ${ }^{26,45}$ In the study by Frexia et al, analyses showed that direct stenting did not influence the impact of IPost on the reported end points. ${ }^{11}$ Due to few studies, we could not formally test the importance of direct stenting in IPost.

\section{Strengths and limitations}

Our review was based on a broad literature search, and it seems unlikely that we missed relevant trials. ${ }^{48}$ The trial selection, data extraction, and data assessment were done by two authors, to minimize bias and transcription errors. ${ }^{34}$ The components used for quality assessment are validated and reported to be associated with bias..$^{29,49}$ The major limitations of our study were the quality of trials, small-study effect, heterogeneity, underpowered trials, and the use of SEPs to evaluate IPost. 
Table 7 Meta-analysis of adverse cardiac events of the six individual trials controlling for variability in duration of follow up, using the patient-years model

\begin{tabular}{|c|c|c|c|c|c|c|}
\hline Study considered & IPost & Patient-years & Control & Patients years & $\mathbf{R R}$ & $95 \% \mathrm{Cl}$ \\
\hline \multicolumn{7}{|l|}{ End point: mortality } \\
\hline Lønborg et a $\left.\right|^{45, a}$ & 3 & 70 & I & 68.75 & 2.95 & $0.31-27.64$ \\
\hline Garcia et $\mathrm{al}^{19}$ & $\mathrm{I}$ & 74.8 & $0^{\mathrm{b}}$ & 71.4 & 2.86 & $0.12-69.15$ \\
\hline Hahn et $\mathrm{a}^{25}$ & 13 & 29.15 & 10 & 29.15 & 1.30 & $0.68-2.48$ \\
\hline Limalanathan et $\mathrm{al}^{12}$ & 4 & 39.9 & 2 & 42.99 & 2.15 & $0.42-11.13$ \\
\hline Pooled-effect estimate & & & & & 1.49 & $0.84-2.64$ \\
\hline \multicolumn{7}{|l|}{ End point: reinfarction } \\
\hline Lønborg et $\mathrm{a}^{45, \mathrm{a}}$ & 4 & 70 & I & 68.75 & 3.93 & $0.45-34.27$ \\
\hline Hahn et $\mathrm{al}^{25}$ & 13 & 29.15 & 10 & 29.15 & 1.30 & $0.68-2.48$ \\
\hline Limalanathan et $\mathrm{al}^{12}$ & 4 & 39.9 & 2 & 42.99 & 2.15 & $0.42-11.13$ \\
\hline Pooled-effect estimate & & & & & 1.50 & $0.84-2.67$ \\
\hline \multicolumn{7}{|l|}{ End point: stent occlusion } \\
\hline Lønborg et a $\mathrm{a}^{45, \mathrm{a}}$ & $0^{\mathrm{b}}$ & 70 & 2 & 68.5 & 0.20 & $0.01-4.00$ \\
\hline Hahn et $\mathrm{a}^{25}$ & 7 & 29.15 & 6 & 29.15 & 1.17 & $0.45-3.05$ \\
\hline Limalanathan et al ${ }^{12}$ & 3 & 39.9 & I & 42.99 & 3.23 & $0.35-29.81$ \\
\hline Pooled-effect estimate & & & & & 1.17 & $0.50-2.74$ \\
\hline \multicolumn{7}{|l|}{ End point: CHF } \\
\hline Lønborg et $\mathrm{a}^{45, \mathrm{a}}$ & 7 & 70 & 15 & 68.75 & 0.46 & $0.20-1.05$ \\
\hline Garcia et al ${ }^{19}$ & 2 & 74.8 & 4 & 71.4 & 0.48 & $0.09-2.53$ \\
\hline Hahn et $\mathrm{al}^{25}$ & 2 & 29.15 & 5 & 29.15 & 0.40 & $0.08-1.90$ \\
\hline Dong et $\mathrm{al}^{26}$ & $0^{\mathrm{b}}$ & 2.66 & 2 & 2.50 & 0.19 & $0.01-2.28$ \\
\hline Limalanathan et $\mathrm{al}^{12}$ & 2 & 39.9 & 5 & 42.99 & 0.43 & $0.09-2.09$ \\
\hline Pooled-effect estimate & & & & & 0.43 & $0.23-0.77$ \\
\hline \multicolumn{7}{|c|}{ End point: major adverse events } \\
\hline Elżbieciak et a ${ }^{23}$ & 4 & 18 & 4 & 21 & 1.17 & $0.34-4.01$ \\
\hline Hahn et $\mathrm{al}^{25}$ & 15 & 29.15 & 13 & 29.15 & 1.15 & $0.67-1.97$ \\
\hline Pooled-effect estimate & & & & & 1.16 & $0.7 \mid-1.89$ \\
\hline
\end{tabular}

Notes: a We considered the study by Lønborg et al ${ }^{45}$ with the longest duration of follow up; bthe problem with zero cell, was dealt with by adding 0.5 to the two arms. Abbreviations: CHF, congestive heart failure; $\mathrm{Cl}$, confidence interval; IPost, ischemic postconditioning; RR, relative risk.

\section{Quality of trials and the effect of IPost on biomarkers of myocardial necrosis and LVEF}

Our results concerning the efficacy of IPost compared with usual care on the outcome biomarkers of myocardial necrosis and LVEF were altered when stratifying on

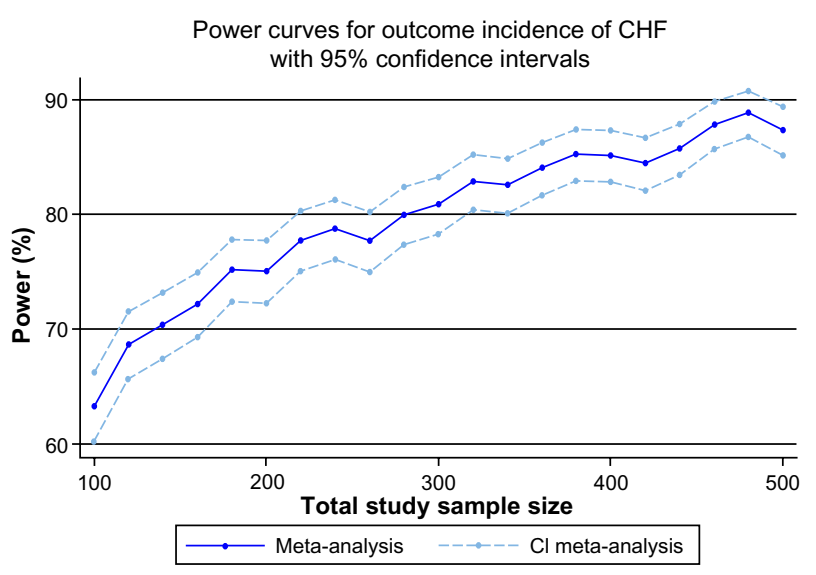

Figure 6 Simulation of power study for a future meta-analysis that could include a future trial with end point, incidence of $\mathrm{CHF}$, using the fixed effect model. Abbreviations: $\mathrm{CHF}$, congestive heart failure; $\mathrm{Cl}$, confidence interval. the components of trial quality. As recommended by the Cochrane Collaboration tools for assessing risk of bias in randomized trials, ${ }^{29}$ we did not use summary scores to identify quality of trials. Instead, we assessed trials methodologically and individually and explored the influence of each on the effect size. Many meta-analyses assess the quality of trials and exclude trials of low quality in sensitivity analysis. When we stratified on the quality of trials, we conclusively found no effect of IPost on markers of myocardial necrosis and LVEF.

\section{IPost and small-study effect for the outcome LVEF}

In the included studies, LVEF was measured by echocardiography or CMR at different time points after PCI, within 7 days or up to 4 months. The differing durations of follow up were investigated by meta-regression and did not alter our conclusion. The change in LVEF before and after IPost would be most interesting for an evaluation of the efficacy of the procedure as opposed to a single estimate of LVEF taken after PCI. However, estimates of LVEF before PCI would be difficult to obtain in a STEMI trial. 
The majority of the trials in our study were small, except for Hahn et $\mathrm{a}^{25}$ and Limalanathan et al. ${ }^{12}$ Because of this, there was a tendency for the small trials to show larger treatment effect. The plausible reason for the effect of intervention to be greater in smaller trials is that differences between small trials and larger trials are accounted for by trial characteristics, as pointed out in our study. An investigation by Hempel et al used Monte Carlo simulation and concluded that larger treatment effects were highlighted in lower quality trials as compared with trials of higher quality. ${ }^{52}$ In our study, the nonbiased trials gave a clear answer for the nonefficacy of IPost as compared with usual care for the end point biomarkers of myocardial necrosis and LVEF.

Unfortunately, in the trials included in our meta-analysis, 13 out of 21 did not have an a priori power evaluation for the primary outcome considered. Some investigators have studied the impact of underpowered trials on metaanalysis results..$^{53}$ In previous meta-analyses of controlled pharmacological trials, effects were $15 \%$ better in underpowered than in adequately powered studies and $12 \%$ better in meta-analyses of controlled nonpharmacological trials. In an empirical approach, the standard error of the intervention effect increased by a median of $11 \%$ when the underpowered studies were omitted, and between-study heterogeneity tended to decrease. The investigators advocated the omission of underpowered trials in a meta-analysis. ${ }^{53}$ When we used this strategy in our meta-analysis, the lack of effect of IPost in the treatment of STEMI was clearly demonstrated.

\section{The problem of heterogeneity due to patient-level and study-level variables in our study}

Meta-regression analyses on patient-level and study-level variables were performed when heterogeneity was present but was not performed when the number of studies was less than ten. Patient-level variables were specified a priori in the protocol of our study, taking into consideration only biologically plausible mechanisms, in order to safeguard against biased conclusions. ${ }^{54}$ Patient-related variables were limited, to avoid potentially false-positive results. Because of power problem and concern, most experts recommend that not more than one covariate for every ten studies be evaluated in a single model/analysis in the meta-regression. ${ }^{55}$ This means that at least 20 studies would be required to run a multivariate meta-regression (two variables addressed at the same time in the metaregression). Our multivariate model was performed on only 15 studies and suffers from power limitation. However, a multivariate analysis has an advantage as it may better control for confounding. On the other hand, it is well known that aggregate variables (patient-related variables) are subject to ecological fallacy and must only be considered as hypothesis generating, ${ }^{56}$ while study-level variables considered in a meta-regression can detect true associations between heterogeneous treatment effects and the study-level variables..$^{57}$

\section{Discrepancies in efficacy of IPost between surrogate endpoints and clinical outcomes}

Our results suggest that IPost has no efficacy compared with usual care with respect to the SEPs infarct size estimated by CMR, biomarkers of myocardial necrosis, LVEF, or ST-segment resolution or on the incidence of adverse cardiac clinical events, with the exception of a reduction in the incidence of CHF during follow up. The number of patients with CHF during follow up was small, and this result must be interpreted with caution. Functional status is often evaluated according to the New York Heart Association classification system. This classification is limited to only four levels and can easily miss clinically important variations if the changes are relatively small in magnitude, and interobserver agreement is often poor. ${ }^{58}$ The consequences of assessing CHF by New York Heart Association class is that there is a probability of misclassification of this end point. In the study by Lønborg et al, ${ }^{45}$ the investigator was blinded to the treatment groups when assessing the functional status of the patient. The nature of this misclassification is nondifferential, ${ }^{59}$ and its effect will dilute the efficacy of IPost on the incidence of CHF. Presumably the true effect of IPost is stronger than the one estimated by this study.

All studies included in our analyses had SEPs as primary outcomes. Infarct size measured by CMR has been shown to be associated with clinical outcome and is probably the most robust SEP to evaluate the effect of new treatment strategies in moderate-size STEMI trials. ${ }^{46,60}$ Some investigators have reported the effect of IPost on myocardial salvage, ie, infarct size as a proportion of area at risk, but there were too few such studies to perform a meaningful meta-analysis using this outcome. However, ultimately, a large-scale, randomized trial with clinical end points is needed to determine the effect of a new treatment principle. In the case of IPost, to the best of our knowledge, only one such study is ongoing, aiming to include 2,000 STEMI patients and including a clinical 
composite primary end point consisting of cardiac death, reinfarction, and heart failure. ${ }^{61}$ Although our data strongly point to a neutral effect of IPost in STEMI, it is conceivable that IPost might affect adverse clinical events by unknown mechanisms operating independently of the mechanisms that determine SEPs. ${ }^{62-64}$

\section{Implications for research}

The quality of reporting in the included trials of our metaanalysis was variable. Future trials should adhere to the methodological standards that reduce possible bias, including concealment of allocation, blinding of treatment groups for outcome assessors, measures to reduce the drop-out rate, and an analysis based on all patients recruited, regardless of the intervention (intention-to-treat strategy). Also, an a priori power analysis is mandatory. Moreover, reports of trials should adhere to generally accepted standards of reporting clinical trials (Consolidated Standards Of Reporting Trials [CONSORT] statements), ${ }^{65}$ and we advocate for continuation of the clinical follow up, in order to find a final answer on the efficacy of IPost on the long-term incidence of CHF during follow up.

\section{Implications for practice}

Trials using SEPs can be misleading and may confuse clinical decisions. RCTs large enough to evaluate effects on clinical outcomes are essential for evidence-based decisions in clinical practice. Our results suggest that further careful trials with appropriate size and clinical end points are necessary before proposing IPost as a supplement to primary PCI in the treatment of STEMI-patients.

\section{Author contribution}

All the authors made substantial contributions to conception and design, acquisition of data, or analysis and interpretation of data. All participated in drafting of the article or revising it critically for important intellectual content, and gave final approval of the version to be published. All are accountable for all aspects of the work, including ensuring that questions related to the accuracy or integrity of any part of the work were appropriately investigated and resolved.

\section{Acknowledgments}

We would like to thank our Medical Librarian, Marie Isachsen, for assisting with the literature search.

\section{Disclosure}

The authors report no conflicts of interest in this work.

\section{References}

1. Steg PG, James SK, Atar D, et al; Task Force on the management of ST-segment elevation acute myocardial infarction of the European Society of Cardiology (ESC). ESC Guidelines for the management of acute myocardial infarction in patients presenting with ST-segment elevation. Eur Heart J. 2012;33(20):2569-2619.

2. Fox KA, Carruthers KF, Dunbar DR, et al. Underestimated and under-recognized: the late consequences of acute coronary syndrome (GRACE UK-Belgian Study). Eur Heart J. 2010;31(22):2755-2764.

3. Yellon DM, Hausenloy DJ. Myocardial reperfusion injury. $N$ Engl J Med. 2007;357(11):1121-1135.

4. Zhao ZQ, Corvera JS, Halkos ME, et al. Inhibition of myocardial injury by ischemic postconditioning during reperfusion: comparison with ischemic preconditioning. Am J Physiol Heart Circ Physiol. 2003;285(2):H579-H588.

5. Staat P, Rifoul G, Piot C, et al. Postconditioning the human heart. Circulation. 2005;112(14):2143-2148.

6. Yang XC, Liu Y, Wang LF, et al. Reduction in myocardial infarct size by postconditioning in patients after percutaneous coronary intervention. J Invasive Cardiol. 2007;19(10):424-430.

7. Thibault H, Piot C, Staat P, et al. Long-term benefit of postconditioning. Circulation. 2008;117(8):1037-1044.

8. Heusch G. Cardioprotection: chances and challenges of its translation to the clinic. Lancet. 2013;381(9861):166-175.

9. Sörensson P, Saleh N, Bouvier F, et al. Effect of postconditioning on infarct size in patients with ST elevation myocardial infarction. Heart. 2010;96(21):1710-1715.

10. Tarantini G, Favaretto E, Marra MP, et al. Postconditioning during coronary angioplasty in acute myocardial infarction: the POST-AMI trial. Int J Cardiol. 2012;162(1):33-38.

11. Freixa X, Bellera N, Ortiz-Pérez JT, et al. Ischaemic postconditioning revisited: lack of effects on infarct size following primary percutaneous coronary intervention. Eur Heart J. 2012;33(1):103-112.

12. Limalanathan S, Andersen GØ, Kløw NE, Abdelnoor M, Hoffmann P, Eritsland J. Effect of ischemic postconditioning on infarct size in patients with ST-elevation myocardial infarction treated by primary PCI results of the POSTEMI (POstconditioning in ST-Elevation Myocardial Infarction) randomized trial. J Am Heart Assoc. 2014;3(2):e000679.

13. Laskey WK. Brief repetitive balloon occlusions enhance reperfusion during percutaneous coronary intervention for acute myocardial infarction: a pilot study. Catheter Cardiovasc Interv. 2005;65(3): 361-367.

14. Ma X, Zhang X, Li C, Luo M. Effect of postconditioning on coronary blood flow velocity and endothelial function and LV recovery after myocardial infarction. J Interv Cardiol. 2006;19(5):367-375.

15. Laskey WK, Yoon S, Calzada N, Ricciardi MJ. Concordant improvements in coronary flow reserve and ST-segment resolution during percutaneous coronary intervention for acute myocardial infarction: a benefit of postconditioning. Catheter Cardiovasc Interv. 2008;72(2):212-220.

16. Lønborg J, Kelbaek H, Vejlstrup N, et al. Cardioprotective effects of ischemic postconditioning in patients treated with primary percutaneous coronary intervention, evaluated by magnetic resonance. Circ Cardiovasc Interv. 2010;3(1):34-41.

17. Xue F, Yang X, Zhang B, et al. Postconditioning in human heart in percutaneous coronary intervention. Clin Cardiol. 2010;33(7):439-444.

18. Lin XM, Zhang ZY, Wang LF, et al. Attenuation of tumor necrosis factor-alpha elevation and improved heart function by postconditioning for 60 seconds in patients with acute myocardial infarction. Chin Med $J$ (Engl). 2010;123(14):1833-1839.

19. Garcia S, Henry TD, Wang YL, et al. Long-term follow-up of patients undergoing postconditioning during ST-elevation myocardial infarction. J Cardiovasc Transl Res. 2011;4(1):92-98.

20. Liu TK, Mishra AK, Ding FX. [Protective effect of ischemia postconditioning on reperfusion injury in patients with ST-segment elevation acute myocardial infarction]. Zhonghua Xin Xue Guan Bing Za Zhi. 2011;39(1):35-39. Chinese. 
21. Zhao CM, Yang XJ, Yang JH, et al. Effect of ischaemic postconditioning on recovery of left ventricular contractile function after acute myocardial infarction. $J$ Int Med Res. 2012;40(3):1082-1088.

22. Thuny F, Lairez O, Roubille F, et al. Post-conditioning reduces infarct size and edema in patients with ST-segment elevation myocardial infarction. J Am Coll Cardiol. 2012;59(24):2175-2181.

23. Elżbieciak M, Wita K, Grabka M, et al. Effect of postconditioning on infarction size, adverse left ventricular remodeling, and improvement in left ventricular systolic function in patients with first anterior ST-segment elevation myocardial infarction. Pol Arch Med Wewn. 2013;123(6):268-276.

24. Dwyer NB, Mikami Y, Hilland D, et al. No cardioprotective benefit of ischemic postconditioning in patients with ST-segment elevation myocardial infarction. J Interv Cardiol. 2013;26(5):482-490.

25. Hahn JY, Song YB, Kim EK, et al. Ischemic postconditioning during primary percutaneous coronary intervention: the effects of postconditioning on myocardial reperfusion in patients with ST-segment elevation myocardial infarction (POST) randomized trial. Circulation. 2013;128(17):1889-1896.

26. Dong M, Mu N, Guo F, et al. The beneficial effects of postconditioning on no-reflow phenomenon after percutaneous coronary intervention in patients with ST-elevation acute myocardial infarction. J Thromb Thrombolysis. 2014;38(2):208-214.

27. Roubille F, Mewton N, Elbaz M, et al. No post-conditioning in the human heart with thrombolysis in myocardial infarction flow 2-3 on admission. Eur Heart J. 2014;35(25):1675-1682.

28. Mewton N, Thibault H, Roubille F, et al. Postconditioning attenuates no-reflow in STEMI patients. Basic Res Cardiol. 2013;108(6):383.

29. Higgins JP, Altman DG, Gøtzsche PC, et al; Cochrane Bias Methods Group; Cochrane Statistical Methods Group. The Cochrane Collaboration's tool for assessing risk of bias in randomized trials. $B M J$. 2011;343:d5928.

30. Bell ML, Kenward MG, Fairclough DL, Horton NJ. Differential dropout and bias in randomised controlled trials: when it matters and when it may not. BMJ. 2013;346:e8668.

31. Johanson P, Jernberg T, Gunnarsson G, Lindahl B, Wallentin L, Dellborg M. Prognostic value of ST-segment resolution-when and what to measure. Eur Heart J. 2003;24(4):337-345.

32. DerSimonian R, Laird N. Meta-analysis in clinical trials. Control Clin Trials. 1986;7(3):177-188.

33. Higgins JP, Thompson SG, Deeks JJ, Altman DG. Measuring inconsistency in meta-anlyses. BMJ. 2003;327(7414):557-560.

34. Egger M, Smith GD, Altman DG, editors. Systematic Reviews in Health Care: Meta-Analysis in Context. London: BMJ Publishing Group; 1995.

35. Sterne JA, Egger M, Smith GD. Systematic reviews in health care: Investigating and dealing with publication and other biases in meta-analysis. BMJ. 2001;323(7304):101-105.

36. Egger M, Davey Smith G, Schneider M, Minder C. Bias in metaanalysis detected by a simple, graphical test. $B M J .1997 ; 315(7109)$ : 629-634.

37. Duval S, Tweedie R. Trim and fill: A simple funnel-plot-based method of testing and adjusting for publication bias in meta-analysis. Biometrics. 2000;56(2):455-463.

38. Idris NRN. Performance of the trim and fill method adjusting for the publication bias in meta-analysis of continuous data. Am J Appl Sci. 2012;9(9):1512-1517.

39. Peters JL, Sutton AJ, Jones DR, Abrams KR, Rushton L. Performance of the trim and fill method in the presence of publication bias and between-study heterogeneity. Stat Med. 2007;26(25):4544-4562.

40. Sutton AJ, Cooper NJ, Jones DR, Lambert PC, Thompson JR, Abrams KR. Evidence-based sample size calculations based upon updated meta-analysis. Stat Med. 2007;26(12):2479-2500.

41. Crowther MJ, Hinchliffe SR, Donald A, Sutton AJ. Simulation-based sample-size calculation for designing new clinical trials and diagnostic test accuracy studies to update an existing meta-analysis. The Stata Journal. 2013;13(3):451-473.
42. Borenstein M, Hegges LV, Higgins JPT, Rothstein HR. Introduction to Meta-Analysis. Chichester: John Wiley \& Sons, Ltd; 2011.

43. Stata.com [homepage on the Internet]. Statacorp LP; 2014. Available from: http://www.stata.com. Accessed July 8, 2014.

44. Moher D, Cook DJ, Eastwood S, Olkin I, Rennie D, Stroup DF. Improving the quality of reports of meta-analyses of randomised controlled trials: the QUOROM statement. Quality of Reporting of Meta-analyses. Lancet. 1999;354(9193):1896-1900.

45. Lønborg J, Holmvang L, Kelbæk H, et al. ST-Segment resolution and clinical outcome with ischemic postconditioning and comparison to magnetic resonance. Am Heart J. 2010;160(6): $1085-1091$

46. Ibrahim T, Bülow HP, Hack1 T, et al. Diagnostic value of contrastenhanced magnetic resonance imaging and single-photon emission computed tomography for detection of myocardial necrosis early after acute myocardial infarction. $J$ Am Coll Cardiol. 2007;49(2): 208-216.

47. Heusch G. Reduction of infarct size by ischaemic post-conditioning in humans: fact or fiction? Eur Heart J. 2012;33(1):13-15.

48. Egger M, Juni P, Bartlett C, Holenstein F, Sterne J. How important are comprehensive literature searches and the assessment of trial quality in systematic reviews? Empirical study. Health Technol Assess. 2003;7(1):1-76

49. Schulz KF, Chalmers I, Hayes RJ, Altman DG. Empirical evidence of bias. Dimensions of methodological quality associated with estimates of treatment effects in controlled trials. JAMA. 1995;273(5): 408-412.

50. Jüni $\mathrm{P}$, Witschi A, Bloch R, Egger M. The hazards of scoring the quality of clinical trials for meta-analysis. JAMA. 1999;282(11): 1054-1060.

51. Moher D, Pham B, Jones A, et al. Does quality of reports of randomised trials affect estimates of intervention efficacy reported in meta-analyses? Lancet. 1998;352(9128):609-613.

52. Hempel S, Miles J, Suttorp MJ, et al. Detection of Associations between Trial Quality and Effect Sizes. Rockville, MD: Agency for Healthcare Research and Quality; 2012.

53. Turner RM, Bird SM, Higgins JP. The impact of study size on meta-analyses: examination of underpowered studies in Cochrane reviews. PLoS One. 2013;8(3):e59202.

54. Berlin JA, Santanna J, Schmid CH, Szczech LA, Feldman HI; Anti-Lymphocyte Antibody Induction Therapy Study Group. Individual patient- versus group-level data meta-regressions for the investigation of treatment effect modifiers: ecological bias rears its ugly head. Stat Med. 2002;21(3):371-387.

55. Higgins JPT, Green S, editors. Cochrane Handbook for Systematic Reviews of Interventions Version 5.1.0. The Cochrane Collaboration; 2011 [updated Mar 2011]. Available from whttp://handbook. cochrane.org/chapter_9/9_6_4_meta_regression.htm. Accessed July 8, 2014.

56. Morgenstern $\mathrm{H}$. Uses of ecologic analysis in epidemiologic research. Am J Public Health. 1982;72(12):1336-1344.

57. Schmid CH, Stark PC, Berlin JA, Landais P, Lau J. Meta-regression detected associations between heterogeneous treatment effects and study-level, but not patient-level, factors. J Clin Epidemiol. 2004;57(7):683-697.

58. Goldman L, Hashimoto B, Cook EF, Loscalzo A. Comparative reproducibility and validity of systems for assessing cardiovascular functional class: advantages of a new specific activity scale. Circulation. 1981;64(6):1227-1234.

59. Kleinbaum DG, Kupper LL, Morgenstern H. Epidemiologic Research. Principles and Quantitative Methods. New York: John Wiley and Sons; 1982.

60. Eitel I, Wöhrle J, Suenkel H, et al. Intracoronary compared with intravenous bolus abciximab application during primary percutaneous coronary intervention in ST-segment elevation myocardial infarction: cardiac magnetic resonance substudy of the AIDA STEMI trial. $J$ Am Coll Cardiol. 2013;61(13):1447-1454. 
61. Rigshospitalet. Danish study of optimal acute treatment of patients with ST-elevation myocardial Infarction DANAMI-3). Available from: http:/ www.clinicaltrials.gov/ct2/show/NCT01435408?term=NCT01435408\% 26rank=1. NLM identifier: NCT01435408. Accessed July 8, 2014.

62. Fleming TR, DeMets DL. Surrogate end point in clinical trials: are we being misled? Ann Intern Med. 1996;125(7):605-613.

63. Baker SG. Surrogate endpoints: wishful thinking or reality? $J$ Natl Cancer Inst. 2006;98(8):502-503.
64. De Gruttola V, Fleming T, Lin DY, Coombs R. Validating surrogate markers - are we being naive? J Infect Dis. 1997;175(2): 237-246.

65. Moher D, Schulz KF, Altman DG. The CONSORT statement: revised recommendations for improving the quality of reports of parallelgroup randomized trials. Lancet. 2001;357(9263):1191-1194.

\section{Publish your work in this journal}

Vascular Health and Risk Management is an international, peerreviewed journal of therapeutics and risk management, focusing on concise rapid reporting of clinical studies on the processes involved in the maintenance of vascular health; the monitoring, prevention and treatment of vascular disease and its sequelae; and the involvement of metabolic disorders, particularly diabetes. This journal is indexed on PubMed Central and MedLine. The manuscript management system is completely online and includes a very quick and fair peer-review system, which is all easy to use. Visit http://www.dovepress.com/ testimonials.php to read real quotes from published authors.

Submit your manuscript here: http://www.dovepress.com/vascular-health-and-risk-management-journal 